\title{
ASYMPTOTIC STABILITY OF RAREFACTION WAVES IN RADIATIVE HYDRODYNAMICS*
}

\author{
CHUNJIN LIN $^{\dagger}$
}

\begin{abstract}
In this paper, we study the asymptotic stability of rarefaction waves for solutions to a one-dimensional radiative hydrodynamic system which couples hyperbolic-elliptic equations. We assume that the initial data tend to constant states at $x= \pm \infty$, respectively, and that the corresponding Riemann problem for the compressible Euler equations admits a continuous rarefaction wave solution with small strength. If the initial perturbation is small, the solution is proved to tend to the rarefaction wave as $t \rightarrow+\infty$. The proof is based on the $L^{2}$-energy method and elliptic estimates.
\end{abstract}

Key words. Asymptotic stability, rarefaction wave, hyperbolic-elliptic coupled system.

AMS subject classifications. 35M10, 35Q35.

\section{Introduction}

We consider a system of PDEs describing astrophysical flows, where a gas interacts with radiation through energy exchanges. The evolution of the gas is governed by the following system of equations, written in Lagrangian coordinates:

$$
\left\{\begin{array}{l}
v_{t}-u_{x}=0, \\
u_{t}+p_{x}=0, \\
\left(e+u^{2} / 2\right)_{t}+(p u+q)_{x}=0,
\end{array}\right.
$$

where $v>0, u$, and $e>0$ represent the specific volume, the velocity and the specific energy of the gas, respectively, $p$ is the pressure and $q$ denotes the radiative heat flux. The radiative heat flux $q$ satisfies the following elliptic equation, still written in Lagrangian coordinates:

$$
-\left(\frac{q_{x}}{v}\right)_{x}+v q+\left(\theta^{4}\right)_{x}=0
$$

where $\theta>0$ is the absolute temperature of the gas. System (1.1)-(1.2) can be formally derived by asymptotic arguments, starting from a more complete system involving a kinetic equation for the specific intensity of radiation; see the appendix of [9] and the references therein. We also refer to $[15,22]$ for the physical background.

In (1.1) and (1.2), $v, u, \theta$ and $q$ are chosen as independent variables. The pressure $p$ and the specific internal energy $e$ are related to $v$ and $\theta$ by

$$
p(v, \theta)=\frac{R \theta}{v}, \quad e=\frac{R}{\gamma-1} \theta:=c_{v} \theta,
$$

where $R$ is the perfect gas constant, $\gamma>1$ is the adiabatic constant, and $c_{v}=R /(\gamma-1)$ is the specific heat at constant volume.

\footnotetext{
${ }^{*}$ Received: February 3, 2010; accepted (in revised version): June 3, 2010. Communicated by Song Jiang.

${ }^{\dagger}$ Department of Mathematics, College of Sciences, Hohai University, Nanjing 210098, Jiangsu, China (cjlin@hhu.edu.cn).

Supported in part by the Fundamental Research Funds for the Central Universities (No. B1020275), the Natural Science Foundation of Hohai University (No. 2008432811) and the Nation Natural Science Foundation of China (No. 10926162, No.10871059).
} 
In this paper we are interested in the asymptotic stability of the rarefaction waves. To this end it is convenient to work with the equation for the specific entropy $s$. The second law of thermodynamics asserts that

$$
\theta \mathrm{d} s=\mathrm{d} e+p \mathrm{~d} v .
$$

From (1.3) the expression of the specific entropy $s$ can be obtained: we have $A \exp \frac{s}{c_{v}}=$ $p(v)^{\gamma}$ with $A$ a constant. The pressure $p$, the temperature $\theta$, and the internal energy $e$ can be viewed as functions of $v$ and $s$ :

$$
p=\tilde{p}(v, s), \quad \theta=\tilde{\theta}(v, s), \quad e=\tilde{e}(v, s) .
$$

Note that Assumption (1.3) yields many algebraic simplifications, but we believe that our results still hold for a general pressure law satisfying the usual requirements of thermodynamics. In variables of $v, u$, and $s$, system (1.1) reads

$$
\left\{\begin{array}{l}
v_{t}-u_{x}=0 \\
u_{t}+\tilde{p}(v, s)_{x}=0 \\
s_{t}+\frac{q_{x}}{\tilde{\theta}(v, s)}=0
\end{array}\right.
$$

In what follows we study the coupled system (1.2), (1.5). This system is completed by imposing the initial data

$$
(v, u, s)(0, x)=\left(v_{0}, u_{0}, s_{0}\right)(x) \rightarrow\left(v_{ \pm}, u_{ \pm}, s_{ \pm}\right), \text {as } x \rightarrow \pm \infty,
$$

where $v_{ \pm}>0, u_{ \pm}$, and $s_{ \pm}$are constants which can be connected by a rarefaction wave. Since we focus on the stability of rarefaction waves, throughout this paper we assume that $s_{+}=s_{-}=\bar{s}$.

Next we introduce an approximation for our system (1.2), (1.5) by neglecting the derivatives $q_{x}$ and $q_{x x}$, i.e.:

$$
\left\{\begin{array}{l}
v_{t}-u_{x}=0 \\
u_{t}+\tilde{p}(v, s)_{x}=0 \\
s_{t}=0
\end{array}\right.
$$

We can still associate to the solution of (1.7) the following quantity:

$$
q=-\left(\tilde{\theta}^{4}(v, s)\right)_{x} / v .
$$

Note that system (1.7) is the usual compressible Euler system. There are two families of rarefaction waves for (1.7) which are solutions of the compressible Euler Equation (1.7) with the Riemann data

$$
(v, u, s)(0, x)=\left(v_{0}^{R}, u_{0}^{R}, s_{0}^{R}\right)(x)=\left(v_{ \pm}, u_{ \pm}, \bar{s}\right), \text { for } x \gtrless 0 .
$$

For illustration, we only consider the 1-rarefaction wave. In this case, we assume that the asymptotic data satisfy $v_{-}<v_{+}$. The rarefaction wave $\left(V^{R}, U^{R}, S^{R}\right)(t, x)$ is characterized by

$$
\left\{\begin{array}{l}
\lambda_{1}\left(V^{R}(t, x), \bar{s}\right)=-\sqrt{-\tilde{p}_{v}\left(V^{R}(t, x), \bar{s}\right)}=w^{R}(t, x), \\
S^{R}(t, x)=\bar{s} \\
U^{R}(t, x)=u_{ \pm}-\int_{v_{ \pm}}^{V^{R}(t, x)} \lambda_{1}(z, s) \mathrm{d} z, \\
\lambda_{1 x}\left(V^{R}(t, x), S^{R}(t, x)\right)>0,
\end{array}\right.
$$


where $\lambda_{1}(v, s)$ is the first characteristic speed of the compressible Euler system (1.7) and $w^{R}(t, x)$ is the unique solution to the following Riemann equation

$$
\left\{\begin{array}{l}
w_{t}^{R}+w^{R} w_{x}^{R}=0, \\
w^{R}(0, x)=w_{0}^{R}(x)=w_{ \pm}:=\lambda_{1}\left(v_{ \pm}, \bar{s}\right), \text { for } x \gtrless 0 .
\end{array}\right.
$$

Since $w_{-}<w_{+}$, system (1.10) admits a unique continuous solution which will be given by $(2.3)$ in $\S 2$.

Also associated with the rarefaction wave $\left(V^{R}, U^{R}, S^{R}, \Theta^{R}\right)(t, x)$, the radiative flux $Q^{R}$ is defined by the equality (1.8) with $\tilde{\theta}(v, s)$ replaced by $\Theta^{R}=\tilde{\theta}\left(V^{R}, S^{R}\right)$. The case for the 3-rarefaction wave can be discussed similarly. For more details about the rarefaction wave of compressible Euler equations, we refer to $[20,19,1]$.

Under the above preparation, our asymptotic stability result on the rarefaction wave $\left(V^{R}, U^{R}, S^{R}\right)(t, x)$ is stated in the following theorem.

THEOREM 1.1. Let $\left(V^{R}, U^{R}, S^{R}\right)(t, x)$ be the 1-rarefaction wave solution to the Riemann problem of the compressible Euler system (1.7), (1.9). Assume that the initial data $\left(v_{0}, u_{0}, s_{0}\right)(x)$ of the Cauchy problem (1.2), (1.5), (1.6) satisfy

$$
\begin{aligned}
& \left(v_{0}(x)-v_{ \pm}, u_{0}(x)-u_{ \pm}\right) \in L^{2}\left(\mathbb{R}_{ \pm}\right), \\
& \left(\partial_{x} v_{0}(x), \partial_{x} u_{0}(x)\right) \in H^{1}(\mathbb{R}) .
\end{aligned}
$$

Let $(V, U, \Theta)(t, x)$ denote the smooth approximation of the rarefaction wave constructed by (2.2) in §2, which satisfies the classical Euler system (1.7). We suppose also that

$$
\left\|\left(v_{0}(x)-V(0, x), u_{0}(x)-U(0, x), s_{0}(x)-\bar{s}\right)\right\|_{H^{2}(\mathbb{R})}
$$

is sufficiently small. Then the Cauchy problem (1.5)-(1.6) admits a unique global smooth solution $(v, u, s, q)(t, x)$ satisfying

$$
\lim _{t \rightarrow+\infty} \sup _{x \in \mathbb{R}}\left\{\left|\left(v(t, x)-V^{R}(t, x), u(t, x)-U^{R}(t, x), s(t, x)-\bar{s}\right)\right|\right\}=0 .
$$

REMARK 1.2. Theorem 1.1 states the stability of weak rarefaction waves. In fact, $V(0, x)$ defined in $\S 2$, satisfies

$$
\left\|V(0, x)-v_{ \pm}\right\|_{L^{2}\left(\mathbb{R}_{ \pm}\right)}=O(1) \frac{\left|v_{+}-v_{-}\right|}{\varepsilon},
$$

where $\varepsilon>0$ is a parameter intended to be small. Thus the assumption about the initial perturbation suggests $\left|v_{+}-v_{-}\right|=o(\varepsilon)$. In the same way, $\left|u_{+}-u_{-}\right|=o(\varepsilon)$.

Concerning the large-time behavior of the solutions to the problem (1.1)-(1.2), Kawashima, Nikkuni and Nishibata in [5] showed the global in time existence of solutions in the neighborhood of a constant state in Eulerian coordinates. The situation when the data leads to a shock has been investigated recently in [9], where the existence of a shock profile is established and in [10], where the stability of the shock profile is analyzed. In these results, a smallness assumption on the strength of the shock in needed (by contrast to the scalar case dealt with in [7]). In [10], it is proved that the solution to the problem (1.1), (1.2) approaches the travelling wave of the shock profile (existence result were obtained in [9]):

$$
(V, U, \Theta, Q)(t, x)=(V, U, \Theta, Q)(x-\sigma t),
$$


which is the exact solution of (1.1), (1.2) satisfying $(V, U, \Theta, Q)( \pm \infty)=\left(v_{ \pm}, u_{ \pm}, \theta_{ \pm}, 0\right)$, where $\sigma$ is the constant called the speed of shock. In the case of 1-shock, the asymptotic data verify $v_{-}>v_{+}$, while here for a 1 -rarefaction wave we study the case $v_{-}<v_{+}$.

Such a problem of the stability of the rarefaction wave has been well studied for viscous regularization of hyperbolic systems and the relaxation approximation of conservation laws. For example, the stability of the rarefaction wave for the compressible Navier-Stokes equations has been studied in $[11,4,16]$, and in $[13,14]$ the authors proved the stability of the rarefaction wave for the isentropic flow. For the relaxation approximation of conservation laws, we refer to [12, 23, 17]. We also mention that the vanishing viscosity limit to the rarefaction waves for the Navier-Stokes equations was studied in [2]. By introducing a scaling argument, the problem reduces to the stability of a rarefaction wave for the compressible Navier-Stokes equations with non-smooth initial data.

In $[8,18]$, the stability of the rarefaction wave for a simplified model of radiating gas dynamics has been proved. This simple model, which can be seen as a prototype for discussing the coupled system (1.1), (1.2) is a Burgers-type equation coupled with a linear elliptic equation for the radiation, so that the equation for the gas is scalar. Here, inspired by $[16,11]$, we wish to deal with the full system of hydrodynamic equations coupled with a nonlinear elliptic equation.

The content of this paper is as follows. In $\S 2$, following $[11,4,16]$, we define a smooth approximation of the rarefaction wave solutions and give some properties of this approximation. Then we reformulate system (1.1), (1.2) by considering the perturbation from the smooth approximation of the rarefaction wave and state the stability result for the reformulated system. That stability result is proved in $\S 3$ by the standard energy method combined with elliptic estimates. Notice that these elliptic estimates are crucial and make the analysis different from the case of compressible Navier-Stokes, as discussed in $[11,4,16]$.

In the rest of this paper, we use $C$ or $O(1)$ to denote a generic positive constant which is independent of $t$ and $x . H^{l}(l>0)$ denotes the usual Sobolev space with the norm $\|\cdot\|_{l}$. Let $\|\cdot\|$ denote the usual $L^{2}$ norm, and $|\cdot|_{p}$ denote the $L^{p}$ norm. Note that $\|\cdot\|_{0}=\|\cdot\|=|\cdot|_{2}$.

\section{Smooth approximation and the reformulation problem}

Following $[4,11,16]$, to prove the stability result, we make use of a smooth approximation of the 1-rarefaction wave $\left(V^{R}, U^{R}, S^{R}\right)(t, x)$. Given a small but fixed constant $\varepsilon>0$, let $w(x, t)$ be the unique global smooth solution to the Cauchy problem:

$$
\left\{\begin{array}{l}
w_{t}+w w_{x}=0, \\
w(0, x)=w_{0}(x):=\frac{w_{-}+w_{+}}{2}+\frac{w_{+}-w_{-}}{2} \tanh (\varepsilon x),
\end{array}\right.
$$

where $w_{ \pm}=\lambda_{1}\left(v_{ \pm}, \bar{s}\right)$. Then, $V(t, x), U(t, x), S(t, x)$ and $\Theta(t, x)$ are defined by

$$
\left\{\begin{array}{l}
\lambda_{1}(V(t, x), \bar{s})=-\sqrt{-\tilde{p}_{v}(V(t, x), \bar{s})}=w(t, x), \\
U(t, x)=u_{ \pm}-\int_{v_{ \pm}}^{V(t, x)} \lambda_{1}(z, s) \mathrm{d} z, \\
S(t, x)=\bar{s}, \\
\Theta(t, x)=\tilde{\theta}(V(t, x), \bar{s}),
\end{array}\right.
$$

and $Q(t, x)$ is defined by

$$
Q(t, x)=-\frac{\left(\Theta^{4}(t, x)\right)_{x}}{V(t, x)}
$$


In fact $w(t, x)$ is a good approximation of $w^{R}(t, x)$, the continuous solution of the Riemann problem (1.10); see [13, 21]. Since $w_{-}<w_{+}<0, w^{R}(t, x)=w^{R}\left(\frac{x}{t}\right)$ can be given by

$$
w^{R}(\xi)= \begin{cases}w_{-}, & \xi<w_{-} \\ \xi, & w_{-} \leq \xi \leq w_{+} \\ w_{+}, & \xi>w_{+}\end{cases}
$$

We shall see that $w(t, x)$ fulfills some regularity proprieties. Consequently, $(V, U, \Theta)$ is a good approximation of the 1-rarefaction wave $\left(V^{R}, U^{R}, \Theta^{R}\right)$ (see [13, 21]), and we will say that $(V, U, \Theta, S)$ is the smooth approximation of the rarefaction wave. The estimates on the smooth rarefaction wave are obtained through the properties of $w(t, x)$, which is the unique solution to the Cauchy problem (2.1). Since the initial data $w_{0}(x)$ are strictly increasing, and by the characteristic method, we have the following properties $[4,16]$ :

Lemma 2.1. If $w_{-}<w_{+}$, then the Cauchy problem (2.1) has a unique global solution $w(t, x)$ satisfying

1. $w_{-}<w(t, x)<w_{+}$and $w_{x}(t, x)>0$, for all $(t, x) \in \mathbb{R}^{+} \times \mathbb{R}$.

2. For any $p(1 \leq p \leq \infty)$, there exists a constant $C_{p}$, such that

$$
\begin{aligned}
\left|w_{x}(t)\right|_{p} & \leq C_{p} \min \left\{\tilde{w} \varepsilon^{1-\frac{1}{p}}, \tilde{w}^{\frac{1}{p}} t^{-1+\frac{1}{p}}\right\}, \\
\left|\partial_{x}^{l} w(t)\right|_{p} & \leq C_{p} \min \left\{\tilde{w} \varepsilon^{l-\frac{1}{p}}, \tilde{w}^{l-1-\frac{1}{p}} t^{-1}\right\}, \quad l \geq 2,
\end{aligned}
$$

where $\tilde{w}=\left|w_{-}-w_{+}\right|$.

3. $\lim _{t \rightarrow+\infty} \sup _{x \in \mathbb{R}}\left|w(t, x)-w^{R}\left(\frac{x}{t}\right)\right|=0$.

According to $(2.2)$, the functions $(V, U, S, \Theta)(t, x)$ defined by $w(t, x)$ are globally well-posed and smooth. Furthermore, we check that $(V, U, S, \Theta)(t, x)$ verify

$$
\left\{\begin{array}{l}
V_{t}-U_{x}=0, \\
U_{t}+p(V, \Theta)_{x}=0, \\
S_{t}=0 \\
c_{v} \Theta_{t}+p(V, \Theta) U_{x}=0 .
\end{array}\right.
$$

Due to Lemma 2.1, $(V, U, \Theta, S)(t, x)$ also have the following properties:

LEMma 2.2. Let us denote $\delta=\left|v_{-}-v_{+}\right|+\left|u_{-}-u_{+}\right|$. The smooth functions $(V, U, \Theta, S)(t, x)$ constructed above have the following properties:

1. $V_{t}=U_{x}>0$, for all $(t, x) \in \mathbb{R}^{+} \times \mathbb{R}$.

2. For any $p(1 \leq p \leq \infty)$, there exists a constants $C_{p}$, such that

$$
\begin{aligned}
\left|V_{x}, U_{x}, \Theta_{x}\right|_{p} & \leq C_{p} \min \left\{\delta \varepsilon^{1-\frac{1}{p}}, \delta^{\frac{1}{p}} t^{-1+\frac{1}{p}}\right\}, \\
\left|\partial_{x}^{l}(V, U, \Theta)\right|_{p} & \leq C_{p} \min \left\{\delta \varepsilon^{l-\frac{1}{p}}, \varepsilon^{l-1-\frac{1}{p}} t^{-1}\right\}, \quad l \geq 2 .
\end{aligned}
$$

3. $\left|(V, U, \Theta)_{t}(t, x)\right| \leq C\left|(V, U, \Theta)_{x}(t, x)\right|$.

4. $\lim _{t \rightarrow+\infty} \sup _{x \in \mathbb{R}}\left|(V, U, S, \Theta)(t, x)-\left(V^{R}, U^{R}, S^{R}, \Theta^{R}\right)(t, x)\right|=0$. 
Under the above preparations, let us state the reformation problem. To this end we set

$$
(\varphi, \psi, \phi, \xi, z)(t, x)=(v-V, u-U, \theta-\Theta, s-\bar{s}, q-Q)(t, x),
$$

and recall that $Q(t, x)=-\left(\Theta^{4}(t, x)\right)_{x} / V(t, x)$. Here $(\varphi, \psi, \phi, z)(t, x)$ represents the perturbation of the rarefaction wave, and solves

$$
\left\{\begin{array}{l}
\varphi_{t}-\psi_{x}=0 \\
\psi_{t}+[p(v, \theta)-p(V, \Theta)]_{x}=0 \\
c_{v} \phi_{t}+[p(v, \theta)-p(V, \Theta)] U_{x}+p(v, \theta) \psi_{x}+z_{x}=\left(\frac{\left(\Theta^{4}\right)_{x}}{V}\right)_{x} \\
v z-\left(\frac{z_{x}}{v}\right)_{x}+\left(\theta^{4}-\Theta^{4}\right)_{x}=\frac{\left(\Theta^{4}\right)_{x}}{V} \varphi-\left\{\left(\frac{\left(\Theta^{4}\right)_{x}}{V}\right)_{x} \frac{1}{v}\right\}_{x},
\end{array}\right.
$$

with the initial data

$$
(\varphi, \psi, \phi)(0, x)=\left(\varphi_{0}, \psi_{0}, \phi_{0}\right)(x):=\left(v_{0}(x)-V(0, x), u_{0}-U(0, x), \theta_{0}(x)-\Theta(0, x)\right) .
$$

In what follows we choose $\varphi, \psi, \phi$ and $z$ as independent variables. For any $T>0$, we define the functional space:

$$
X(0, T)=\left\{(\varphi, \psi, \phi, z)(t, x) \mid \begin{array}{c}
(\varphi, \psi, \phi) \in L^{\infty}\left(0, T ; H^{2}(\mathbb{R})\right) \\
\left(\varphi_{x}, \psi_{x}, \phi_{x}\right) \in L^{2}\left(0, T ; H^{1}(\mathbb{R})\right), \\
z \in L^{\infty}\left(0, T ; H^{3}(\mathbb{R})\right) \cap L^{2}\left(0, T ; H^{3}(\mathbb{R})\right)
\end{array}\right\} .
$$

Thus we are going to prove the following result:

Theorem 2.3. Under the hypotheses stated in Theorem 1.1, the Cauchy problem (2.4) admits a unique global solution $(\varphi, \psi, \phi, z) \in X(0,+\infty)$. Furthermore the solution satisfies the following uniform estimate:

$$
\begin{aligned}
\|(\varphi, \psi, \phi)(t) & \left\|_{2}^{2}+\right\| z(t)\left\|_{3}^{2}+\int_{0}^{t}\right\| V_{t}^{1 / 2}(\tau)(\varphi, \phi)(\tau) \|^{2} \mathrm{~d} \tau \\
& +\int_{0}^{t}\left(\left\|\left(\varphi_{x}, \psi_{x}, \phi_{x}\right)(\tau)\right\|_{1}^{2}+\|z(\tau)\|_{3}^{2}\right) \mathrm{d} \tau \leq C\left(\left\|\left(\varphi_{0}, \psi_{0}, \phi_{0}\right)(t)\right\|_{2}^{2}+\varepsilon^{\frac{1}{4}}\right),
\end{aligned}
$$

for any $t>0$.

The global existence follows by combining a local result and the a priori estimate (2.6). The local existence of solutions of such a hyperbolic-elliptic system is proved by the standard iteration method; we omit the details and refer to [5, 6]. Hence, to complete the proof of Theorem 2.3, the crucial step is to show the a priori estimate (2.6). To this end, we set

$$
N(t)^{2}=\sup _{\tau \in[0, t]}\left(\|(\varphi, \psi, \phi)(\tau)\|_{2}^{2}+\|z(\tau)\|_{3}^{2}\right) .
$$


Proposition 2.4. Let $T>0$ and let $(\varphi, \psi, \phi, z) \in X(0, T)$ be the unique solution to system (2.4), (2.5). Then there exists a constant $\hat{\varepsilon}$ independent of $T$ such that if $N(T) \leq \hat{\varepsilon}$, and $\varepsilon \leq \hat{\varepsilon}$, then the estimate

$$
\begin{aligned}
\|(\varphi, \psi, \phi)(t) & \left\|_{2}^{2}+\right\| z(t)\left\|_{3}^{2}+\int_{0}^{t}\right\| V_{t}^{1 / 2}(\tau)(\varphi, \phi)(\tau) \|^{2} \mathrm{~d} \tau \\
& +\int_{0}^{t}\left(\left\|\left(\varphi_{x}, \psi_{x}, \phi_{x}\right)(\tau)\right\|_{1}^{2}+\|z(\tau)\|_{3}^{2}\right) \mathrm{d} \tau \leq C\left(\left\|\left(\varphi_{0}, \psi_{0}, \phi_{0}\right)(t)\right\|_{2}^{2}+\varepsilon^{\frac{1}{4}}\right)
\end{aligned}
$$

holds for any $t \in[0, T]$, where the constant $C$ does not depend on $T$.

It follows from Proposition 2.4 and the standard continuation argument (c.f [3]) that we get the proof of Theorem 2.3. The proof of Proposition 2.4 is given in the next section, and is based on the energy method combined with the elliptic estimates.

\section{A priori estimate}

In this section we will use the energy method to the perturbation system. Firstly we show the $L^{\infty}\left(H^{2}\right)$ estimate of the fluid perturbation variables $(\varphi, \psi, \phi)$. Notice that linearizing the equations around the smooth approximation of the rarefaction wave is no longer a valid approach. As pointed in [10], this is because the dissipation introduced by the elliptic equation is not strong enough to control the linearization errors. However, the standard symmetrization of the quasi-linear form of the perturbation system (2.4) works, as we shall see below. Following [3], we then give the $L^{2}\left(H^{1}\right)$ estimate of the derivatives of the fluid perturbation variables $(\varphi, \psi)$. Eventually the elliptic inequalities will be given by using the elliptic equation.

3.1. $L^{\infty}\left(H^{2}\right)$ estimate. In this paragraph we wish to prove the following proposition.

Proposition 3.1. Under the assumptions stated in Proposition 2.4, there holds

$$
\begin{aligned}
& \|(\varphi, \psi, \phi)(t)\|_{2}^{2}+\int_{0}^{t}\left\{\|z(\tau)\|_{3}^{2}+\left\|\sqrt{V_{t}(\tau)}(\varphi, \phi)(\tau)\right\|^{2}\right\} \mathrm{d} \tau \\
\leq & C\left(N(0)^{2}+\varepsilon^{1 / 4}+(N(t)+\varepsilon) \int_{0}^{t}\left\|\left(\varphi_{x}, \psi_{x}, \phi_{x}\right)(\tau)\right\|_{1}^{2} \mathrm{~d} \tau\right) .
\end{aligned}
$$

We split the proof of Proposition 3.1 into two lemmata. Firstly we prove the following

Lemma 3.2. Under the assumptions stated in Proposition 2.4, there holds

$$
\begin{aligned}
& \|(\varphi, \psi, \phi)(t)\|^{2}+\int_{0}^{t}\left\{\left\|\sqrt{V_{t}(\tau)}(\varphi, \phi)(\tau)\right\|^{2}+\|z(\tau)\|_{1}^{2}\right\} \mathrm{d} \tau \\
\leq & C\left(\left\|\left(\varphi_{0}, \psi_{0}, \phi_{0}\right)\right\|^{2}+N(t) \int_{0}^{t}\left\|\left(\varphi_{x}, \phi_{x}\right)(\tau)\right\| \mathrm{d} \tau+\varepsilon^{1 / 4}\right) .
\end{aligned}
$$

Proof. Following $[16,11]$, we introduce the normalized entropy $\eta(\varphi, \psi, \phi, z)$ around the smooth rarefaction $(V, U, \Theta)$ as

$$
\begin{aligned}
\eta(\varphi, \psi, \phi, z)=e( & V+\varphi, \Theta+\phi)+\frac{1}{2}(U+\psi)^{2}-\left[e(V, \Theta)+\frac{1}{2} U^{2}\right] \\
- & {[-p(V, \Theta) \varphi+U \psi+\Theta(s-S)] }
\end{aligned}
$$


and notice that $\tilde{e}_{v}(v, s)=-p(v, \theta), \tilde{e}_{s}(v, s)=\theta$. It is easy to show that $\eta$ satisfies the following equality:

$$
\begin{gathered}
\eta_{t}(\varphi, \psi, \phi, z)+\frac{v z^{2}+z_{x} / v}{4 \theta \Theta^{3}}+\{\cdots\}_{x} \\
+\left[p(v, \theta)-p(V, \Theta)+\frac{p(V, \Theta)}{V} \varphi-\frac{p(V, \Theta}{c_{v}} \phi\right] U_{x}=\sum_{i=1}^{3} \mathcal{H}_{i},
\end{gathered}
$$

where the functions $\mathcal{H}_{i}$ are defined as

$$
\begin{aligned}
& \mathcal{H}_{1}=-\frac{\phi z}{\theta^{2}} \Theta_{x}+\frac{\varphi z}{4 \theta \Theta^{3}}\left(\Theta^{4}\right)_{x}-\frac{\phi z}{4 \theta \Theta^{3}}\left(4 \Theta^{3}\right)_{x}-\frac{z z_{x}}{v}\left(\frac{1}{4 \Theta^{3}}\right)_{x}, \\
& \mathcal{H}_{2}=\left(\frac{\left(\Theta^{4}\right)_{x}}{V}\right)_{x}\left[\frac{\phi}{\theta}+\frac{1}{v}\left(\frac{z}{4 \theta \Theta^{3}}\right)_{x}\right] \\
& \mathcal{H}_{3}=-\frac{z \phi \phi_{x}}{\theta^{2}}-\left(6 \Theta^{2} \phi^{2}+4 \Theta \phi^{3}+\phi^{4}\right)_{x} \frac{z}{4 \theta \Theta^{3}} .
\end{aligned}
$$

Here and after, $\{\cdots\}_{x}$ denotes the terms that will vanish after integrating with respect to $x$. Observe that $\tilde{p}_{v}(v, s)=-p / v, \quad \tilde{p}_{s}(v, \bar{s})=p / c_{v}$. Thus, integrating (3.3) with respect to $t$ and $x$ over $[0, t] \times \mathbb{R}$ yields

$$
\begin{aligned}
& \|(\varphi, \psi, \phi)(t)\|^{2}+\int_{0}^{t}\left\{\left\|\sqrt{V_{t}(\tau)}(\varphi, \phi)(\tau)\right\|^{2}+\|z(\tau)\|_{1}^{2}\right\} \mathrm{d} \tau \\
\leq & C\left(\left\|\left(\varphi_{0}, \psi_{0}, \phi_{0}\right)\right\|^{2}+\int_{0}^{t} \int_{\mathbb{R}} \sum_{i=1}^{3}\left|\mathcal{H}_{i}(\tau, x) \mathrm{d}\right| \mathrm{d} x \mathrm{~d} \tau\right),
\end{aligned}
$$

where we have used the properties of the smooth approximation of the rarefaction wave, Lemma 2.2. Then we estimate all the terms in the right-hand side of (3.4). We start with

$$
\int_{0}^{t} \int_{\mathbb{R}}\left|\mathcal{H}_{1}(\tau, x)\right| \mathrm{d} x \mathrm{~d} \tau \leq C \int_{0}^{t} \int_{\mathbb{R}}\left|\Theta_{x}\right||z||(\varphi, \phi)| \mathrm{d} \tau+\int_{0}^{t} \int_{\mathbb{R}}\left|\Theta_{x} z z_{x}\right| \mathrm{d} x \mathrm{~d} \tau .
$$

Using the Sobolev, Young and Hölder inequalities, the first term in the right-hand side of (3.5) can be estimated by

$$
\begin{aligned}
\int_{0}^{t} \int_{\mathbb{R}}\left|\Theta_{x}\|z\|(\varphi, \phi)\right| \mathrm{d} \tau & \leq C \int_{0}^{t}\|(\varphi, \phi)(\tau)\|^{1 / 2}\left\|\left(\varphi_{x}, \phi_{x}\right)(\tau)\right\|^{1 / 2}\|z(\tau)\|\left\|\Theta_{x}(\tau)\right\| \mathrm{d} \tau \\
& \leq C\left(N(t)^{6} \int_{0}^{t}\left\|\left(\varphi_{x}, \phi_{x}\right)(\tau)\right\|^{2} \mathrm{~d} \tau+\int_{0}^{t}\left\|\Theta_{x}(\tau)\right\|^{4 / 3} \mathrm{~d} \tau\right) \\
& \leq C\left(N(t)^{6} \int_{0}^{t}\left\|\left(\varphi_{x}, \phi_{x}\right)(\tau)\right\|^{2} \mathrm{~d} \tau+\varepsilon^{1 / 4}\right) .
\end{aligned}
$$

Note that in the last inequality we have used Lemma 2.2 and the following inequality

$$
\begin{aligned}
\int_{0}^{t}\left|\partial_{x}^{l} \Theta(\tau)\right|_{p}^{a+b} \mathrm{~d} \tau & \leq \sup _{\tau \in[0, t]}\left|\partial_{x}^{l} \Theta(\tau)\right|_{p}^{a} \int_{0}^{t}\left|\partial_{x}^{l} \Theta(\tau)\right|_{p}^{b} \mathrm{~d} \tau \\
& \leq \sup _{\tau \in[0, t]}\left|\partial_{x}^{l} \Theta(\tau)\right|_{p}^{a} \int_{0}^{t}\left|\partial_{x}^{l} \Theta(\tau)\right|_{p}^{b} \mathrm{~d} \tau \\
& \leq \sup _{\tau \in[0, t]}\left|\partial_{x}^{l} \Theta(\tau)\right|_{p}^{a}\left(\int_{0}^{\varepsilon}\left|\partial_{x}^{l} \Theta(\tau)\right|_{p}^{b} \mathrm{~d} \tau+\int_{\varepsilon}^{+\infty}\left|\partial_{x}^{l} \Theta(\tau)\right|_{p}^{b} \mathrm{~d} \tau\right)
\end{aligned}
$$


Similarly by the Hölder and Cauchy-Schwarz' inequalities and Lemma 2.2, the second term in the right-hand side of (3.5) can be estimated as follows

$$
\int_{0}^{t} \int_{\mathbb{R}}\left|\Theta_{x} z z_{x}\right| \mathrm{d} x \mathrm{~d} \tau \leq \int_{0}^{t}\left|\Theta_{x}(\tau)\right|_{\infty}\left|z z_{x}(\tau)\right|_{1} \mathrm{~d} \tau \leq C \varepsilon \int_{0}^{t}\|z(\tau)\|_{1}^{2} \mathrm{~d} \tau
$$

Thus we have

$$
\int_{0}^{t} \int_{\mathbb{R}}\left|\mathcal{H}_{1}(\tau, x)\right| \mathrm{d} x \mathrm{~d} \tau \leq C\left\{\int_{0}^{t}\left(N(t)^{2}\left\|\left(\varphi_{x}, \phi_{x}\right)(\tau)\right\|^{2}+\varepsilon\|z(\tau)\|_{1}^{2}\right) \mathrm{d} \tau+\varepsilon^{1 / 4}\right\}
$$

where we have used the fact that $N(T)$ is sufficiently small. Next, the term $\mathcal{H}_{2}$ in the right-hand side of (3.4) can be estimated as follows

$$
\begin{aligned}
& \int_{0}^{t} \int_{\mathbb{R}}\left|\mathcal{H}_{2}(\tau, x)\right| \mathrm{d} x \mathrm{~d} \tau \\
\leq & C\left(N(t)^{2} \int_{0}^{t}\left\|\phi_{x}(\tau)\right\|^{2} \mathrm{~d} \tau+\int_{0}^{t}\left|\left(\frac{\left(\Theta^{4}\right)_{x}}{V}\right)_{x}\right|_{1}^{\frac{4}{3}} \mathrm{~d} \tau\right) \\
& +\frac{1}{4} \int_{0}^{t}\|z(\tau)\|_{1}^{2} \mathrm{~d} \tau+C \int_{0}^{t} \int_{R}\left|\frac{1}{v}\left(\frac{\left(\Theta^{4}\right)_{x}}{V}\right)_{x}\right|^{2}\left(\left|\frac{1}{4 \theta \Theta^{3}}\right|^{2}+\left|\left(\frac{1}{4 \theta \Theta^{3}}\right)_{x}\right|^{2}\right) \mathrm{d} x \mathrm{~d} \tau \\
\leq & \frac{1}{4} \int_{0}^{t}\|z(\tau)\|_{1}^{2} \mathrm{~d} \tau+C\left\{N(t)^{2} \int_{0}^{t}\left\|\phi_{x}(\tau)\right\|^{2} \mathrm{~d} \tau+\varepsilon^{1 / 4}\right\},
\end{aligned}
$$

and the term of $\mathcal{H}_{3}$ in the right-hand side of (3.4) can be estimated as follows

$$
\begin{aligned}
\int_{0}^{t} \int_{\mathbb{R}}\left|\mathcal{H}_{3}(\tau, x)\right| \mathrm{d} x \mathrm{~d} \tau & \leq C \int_{0}^{t} \int_{\mathbb{R}}\left(\left|\phi \phi_{x} z\right|+\left|\Theta_{x} z \phi^{2}\right|+\left|\Theta_{x}\right|\left|z \phi \phi_{x}\right|\right) \mathrm{d} x \mathrm{~d} \tau \\
& \leq C N(t) \int_{0}^{t}\left(\|z(\tau)\|^{2}+\left\|\phi_{x}\right\|^{2}+\left\|\sqrt{V_{t}(\tau)} \phi(\tau)\right\|\right) \mathrm{d} \tau
\end{aligned}
$$

Substituting the above estimates of $\mathcal{H}_{i}, i=1,2,3$, into (3.4), and using the fact that $N(t)$ and $\varepsilon$ are sufficiently small, gives the desired estimate (3.2).

To prove Proposition 3.1, it remains to show the $L^{\infty}\left(L^{2}\right)$ estimates of the derivatives of $(\varphi, \psi, \phi)$. We need to use the standard symmetrization of the quasi-linear form of the perturbation system (2.4) to prove the following lemma:

Lemma 3.3. Under the assumptions stated in Proposition 2.4, there holds

$$
\begin{gathered}
\left\|\left(\varphi_{x}, \psi_{x}, \phi_{x}\right)(t)\right\|_{1}^{2}+\int_{0}^{t}\left\|z_{x}(\tau)\right\|_{2}^{2} \mathrm{~d} \tau-C(N(t)+\varepsilon) \int_{0}^{t}\left\|\sqrt{V_{t}(\tau)}(\varphi, \phi)(\tau)\right\|^{2} \mathrm{~d} \tau \\
-C(N(t)+\varepsilon) \int_{0}^{t}\left\|\left(\varphi_{x}, \psi_{x}, \phi_{x}\right)(\tau)\right\|_{1}^{2} \mathrm{~d} \tau \leq C\left(\left\|\left(\varphi_{0}, \psi_{0}, \phi_{0}\right)_{x}\right\|_{1}^{2}+\varepsilon^{1 / 4}\right) .
\end{gathered}
$$


Proof. Following [3, 10], we recast the system (2.4) as

$$
\left\{\begin{array}{l}
\varphi_{t}-\psi_{x}=0 \\
\psi_{t}+\frac{R \phi_{x}}{v}-R \frac{\theta}{(v)^{2}} \varphi_{x}=f_{1}:=R \frac{\varphi \Theta_{x}}{V v}+R \frac{\phi V_{x}}{v^{2}}-R \frac{\varphi^{2}+2 V \varphi}{V^{2} v^{2}} \Theta V_{x} \\
c_{v} \phi_{t}+R \frac{\theta}{v} \psi_{x}+z_{x}-\left(\frac{\left(\Theta^{4}\right)_{x}}{V}\right)_{x}=f_{2}:=[p(v, \theta)-p(V, \Theta)] U_{x} \\
v z-\left(\frac{z_{x}}{v}\right)_{x}+4 \Theta^{3} \phi_{x}+\left(\left(\frac{\left(\Theta^{4}\right)_{x}}{V}\right)_{x} \frac{1}{v}\right)_{x} \\
=f_{3}:=\frac{\varphi\left(\Theta^{4}\right)_{x}}{V}-\left(4 \Theta^{3}\right)_{x} \phi-\left(6 \Theta^{2} \phi^{2}+4 \Theta \phi^{3}+\phi^{4}\right)_{x} .
\end{array}\right.
$$

Then we apply $\partial_{x}$ to the above system, and multiply the resulting equations by $\varphi_{x}, \frac{v^{2}}{R \theta} \psi_{x}, \frac{v^{2}}{R \theta^{2}} \phi_{x}$ and $\frac{v^{2}}{R \theta^{2}} \frac{z_{x}}{4 \Theta^{3}}$, respectively. We get

$$
E_{1}(\varphi, \psi, \phi)_{t}+\{\cdots\}_{x}+E_{2}\left(z_{x}, z_{x x}\right)=\sum_{i=4}^{6} \mathcal{H}_{j}
$$

with

$$
\begin{gathered}
E_{1}(\varphi, \psi, \phi)=\frac{1}{2}\left(\varphi_{x}^{2}+\frac{v^{2}}{R \theta} \psi_{x}^{2}+\frac{1}{c_{v}} \frac{v^{2}}{R \theta^{2}} \phi_{x}^{2}\right) \\
E_{2}\left(z_{x}, z_{x x}\right)=\frac{v^{3}}{4 \Theta^{3} \theta^{2}} z_{x}^{2}+\frac{v x_{x x}}{4 \Theta^{3} \theta^{2}}, \\
\mathcal{H}_{4}=\left(\frac{v}{\theta}\right)_{x} \phi_{x} \psi_{x}+\left(\frac{v^{2}}{\theta^{2}}\right)_{x} \phi_{x} z_{x}-\frac{z_{x} z_{x x}}{v}\left(\frac{v^{2}}{4 R \Theta^{3} \theta^{2}}\right)_{x}+\left(\frac{v}{R \theta}\right)_{t} \psi_{x}^{2}+\frac{c_{v}}{2}\left(\frac{v^{2}}{R \theta^{2}}\right)_{t} \phi_{x}^{2}, \\
\mathcal{H}_{5}=\frac{v^{2}}{R \theta^{2}}\left(\frac{\left(\Theta^{4}\right)_{x}}{V}\right)_{x x} \phi_{x}+\left\{\left(\frac{\left(\Theta^{4}\right)_{x}}{V}\right)_{x} \frac{1}{v}\right\}_{x}\left(\frac{v^{2}}{R \theta^{2}} \frac{z_{x}}{4 \Theta^{3}}\right)_{x}, \\
\mathcal{H}_{6}=\frac{v^{2}}{R \theta} \varphi_{x} \partial_{x} f_{1}+\frac{v^{2}}{R \theta^{2}} \psi_{x} \partial_{x} f_{2}+\frac{z_{x}}{4 \Theta^{3}} \frac{v^{2}}{R \theta^{2}} \partial_{x} f_{3} .
\end{gathered}
$$

We shall estimate the functions $E_{1}, E_{2}$ and $\mathcal{H}_{j}, j=4,5,6$, by using the properties of the smooth rarefaction wave $(V, U, \Theta)$. Firstly, we have

$$
\begin{gathered}
c\left(\varphi_{x}^{2}(t, x)+\psi_{x}^{2}(t, x)+\phi_{x}^{2}(t, x)\right) \leq E_{1}(\varphi, \psi, \phi) \leq C\left(\varphi_{x}^{2}(t, x)+\psi_{x}^{2}(t, x)+\phi_{x}^{2}(t, x)\right) \\
E_{2}\left(z_{x}, z_{x x}\right) \geq c\left(z_{x}^{2}(t, x)+z_{x x}^{2}(t, x)\right)
\end{gathered}
$$

with some positive constants $c$ and $C$. Next the functions $\mathcal{H}_{j}, j=4,5,6$, are estimated as follows:

$$
\begin{gathered}
\mathcal{H}_{4} \leq C(N(t)+\varepsilon)\left|\left(\psi_{x}, \phi_{x}, z_{x}, z_{x x}\right)(t, x)\right|^{2}, \\
\mathcal{H}_{5} \leq C\left(\varepsilon\left|\phi_{x}^{2}(t, x)\right|+\frac{1}{\varepsilon}\left|\left(\frac{\left(\Theta^{4}\right)_{x}}{V}\right)_{x x}\right|^{2}\right)+\frac{1}{4}\left|\left(z_{x}, z_{x x}\right)(t, x)\right|^{2} \\
+C\left|\left\{\left(\frac{\left(\Theta^{4}\right)_{x}}{V}\right)_{x} \frac{1}{v}\right\}_{x}\right|^{2}\left(1+\left|\left(\frac{v^{2}}{4 R \theta^{2} \Theta^{3}}\right)_{x}\right|^{2}\right),
\end{gathered}
$$




$$
\mathcal{H}_{6} \leq C(N(t)+\varepsilon)\left(\left|\sqrt{V_{t}}(\varphi, \phi)(t, x)\right|^{2}+\left|\left(\varphi_{x}, \phi_{x}\right)(t, x)\right|^{2}+\left|z_{x x}(t, x)\right|^{2}\right),
$$

where we have used the Cauchy-Schwarz inequality. Note that $\int_{0}^{t}\left\|\left(\left(\Theta^{4}\right)_{x} / V\right)_{x x}\right\|^{2} \mathrm{~d} \tau \leq$ $C \varepsilon^{3}$. By integrating $(3.8)$ over $[0, t] \times \mathbb{R}$ with respect to variables $t$ and $x$ respectively, and using the above inequalities, we have

$$
\begin{gathered}
\left\|\left(\varphi_{x}, \psi_{x}, \phi_{x}\right)(t)\right\|^{2}+\int_{0}^{t}\left\|z_{x}(\tau)\right\|_{1}^{2} \mathrm{~d} \tau-C(N(t)+\varepsilon) \int_{0}^{t}\left\|\left(\varphi_{x}, \psi_{x}, \phi_{x}\right)(\tau)\right\|^{2} \mathrm{~d} \tau \\
-C(N(t)+\varepsilon) \int_{0}^{t}\left\|\sqrt{V_{t}(\tau)}(\varphi, \phi)(\tau)\right\|^{2} \mathrm{~d} \tau \leq C\left(\left\|\left(\varphi_{0}, \psi_{0}, \psi_{0}\right)_{x}\right\|^{2}+\varepsilon^{1 / 4}\right) .
\end{gathered}
$$

Similarly we apply $\partial_{x x}^{2}$ to the system (3.7), multiply the resulting four equations by $\varphi_{x x}, \frac{v^{2}}{R \theta} \psi_{x x}, \frac{v^{2}}{R \theta^{2}} \phi_{x x}$ and $\frac{z_{x x}}{4 \Theta^{3}} \frac{v^{2}}{R \theta^{2}}$ respectively. Calculating their sums and integrating the resulting equality over $[0, t] \times R$, we can get the following estimate:

$$
\begin{gathered}
\left\|\left(\varphi_{x x}, \psi_{x x}, \phi_{x x}\right)(t)\right\|^{2}+\int_{0}^{t}\left\|z_{x x}(\tau)\right\|_{1}^{2} \mathrm{~d} \tau-C(N(t)+\varepsilon) \int_{0}^{t}\left\|\left(\varphi_{x}, \psi_{x}, \phi_{x}\right)(\tau)\right\|_{1}^{2} \mathrm{~d} \tau \\
-C(N(t)+\varepsilon) \int_{0}^{t}\left\|\sqrt{V_{t}(\tau)}(\varphi, \phi)(\tau)\right\|^{2} \mathrm{~d} \tau \leq C\left(\left\|\left(\varphi_{0}, \psi_{0}, \psi_{0}\right)_{x x}\right\|^{2}+\varepsilon^{1 / 4}\right) .
\end{gathered}
$$

We omit the details here. Using the estimates (3.9) and (3.10), we get the desired estimate (3.6).

With the estimates (3.2) and (3.6), we can easily get the inequality (3.1) and the proof of Proposition 3.1 is complete. To finish the proof of Proposition 2.4 we need now to estimate the last integral in (3.1). We shall start by estimating the derivatives of $\varphi$ and $\psi$ in the $H^{1}$ norm in Section 3.2 and then we will discuss $\phi$ in Section 3.3.

3.2. $L^{2}\left(L^{2}\right)$ estimates. In this paragraph, we show the $L^{2}\left(L^{2}\right)$ estimates of the derivatives of the perturbation variables $\varphi$ and $\psi$. We have the following

Proposition 3.4. Under the assumptions stated in Proposition 2.4, there holds

$$
\begin{aligned}
& \int_{0}^{t}\left\|\left(\varphi_{x}, \psi_{x}\right)(\tau)\right\|_{1}^{2} \mathrm{~d} \tau-C \int_{0}^{t}\left(\left\|\sqrt{V_{t}(\tau)}(\varphi, \phi)(\tau)\right\|^{2}+\left\|\left(\phi_{x}, z_{x}\right)(\tau)\right\|_{1}^{2}\right) \mathrm{d} \tau \\
\leq & C\left(\left\|\left(\varphi_{0}, \psi_{0}, \phi_{0 x}\right)\right\|^{2}+\left\|\left(\varphi, \phi, \psi_{x}\right)(t)\right\|_{1}^{2}\right) .
\end{aligned}
$$

Proof. We rewrite the second and the third equations of the perturbation system (2.4) around the smooth rarefaction wave $(V, U, \Theta)$ :

$$
\left\{\begin{array}{l}
\psi_{t}+\frac{R \phi_{x}}{V}-R \frac{\Theta}{V^{2}} \varphi_{x}=\tilde{f}_{1}:=f_{1}+R \frac{\varphi \phi_{x}}{V v}+R \frac{\phi \varphi_{x}}{v^{2}}-R \frac{\varphi^{2}+2 V \varphi}{V^{2} v^{2}} \Theta \varphi_{x} \\
c_{v} \phi_{t}+R \frac{\Theta}{V} \psi_{x}+z_{x}=\tilde{f}_{2}:=f_{2}+\left(\frac{\left(\Theta^{4}\right)_{x}}{V}\right)_{x}-\frac{R \Theta \varphi}{V v}+\frac{R \varphi}{v}
\end{array}\right.
$$

Multiply the above equations by $-V \varphi_{x}$ and $\frac{2 V^{2}}{R \Theta} \psi_{x}$ respectively, then calculate their sum. We get

$$
\begin{aligned}
& \frac{R \Theta}{V} \varphi_{x}^{2}+V \psi_{x}^{2}+\{\cdots\}_{x}+\left(V \psi_{x} \varphi-c_{v} \frac{2 V}{R \Theta} \phi \psi_{x}\right)_{t}=R \phi_{x} \varphi_{x}-\left[\varphi V_{x}+c_{v}\left(\frac{2 V}{R \Theta} \phi\right)_{x}\right] \psi_{t} \\
& +\psi_{x} V_{t} \varphi+\frac{2 V^{2}}{R \Theta} \psi_{x} z_{x}-c_{v}\left(\frac{2 V^{2}}{R \Theta}\right)_{t} \phi \psi_{x}-\tilde{f}_{1} V \varphi_{x}+\tilde{f}_{2} \frac{2 V^{2}}{R \Theta} \psi_{x}
\end{aligned}
$$


Integrating the above equality over $[0, t] \times \mathbb{R}$ yields

$$
\begin{aligned}
& \int_{0}^{t}\left\|\left(\varphi_{x}, \psi_{x}\right)(\tau)\right\|^{2} \mathrm{~d} \tau \\
\leq & C\left(\left\|\left(\varphi_{0}, \psi_{0}, \phi_{0 x}\right)\right\|^{2}+\left\|\left(\varphi, \phi, \psi_{x}\right)(t)\right\|^{2}+\int_{0}^{t} \int_{\mathbb{R}} \sum_{j=7}^{8}\left|\mathcal{H}_{j}(\tau, x)\right| \mathrm{d} x \mathrm{~d} \tau\right),
\end{aligned}
$$

with

$$
\begin{aligned}
\mathcal{H}_{7}= & R \phi_{x} \psi_{x}+\frac{2 V^{2}}{R \Theta} \psi_{x} z_{x}+\psi_{x} V_{t} \varphi-c_{v} \psi_{x} \phi\left(\frac{2 V^{2}}{R \Theta}\right)_{t} \\
& +\frac{R}{V} \varphi V_{x}\left(\phi_{x}-\frac{\Theta}{V} \varphi_{x}\right)+c_{v}\left(\frac{2 V^{2}}{R \Theta} \phi\right)_{x} \frac{R}{V}\left(\phi_{x}-\frac{\Theta}{V} \varphi_{x}\right), \\
\mathcal{H}_{8}= & -\tilde{f}_{1}\left[(V \varphi)_{x}+c_{v}\left(\frac{2 V^{2} \varphi}{R \Theta} \phi\right)_{x}\right]+\tilde{f}_{2} \frac{2 V^{2}}{R \Theta} \psi_{x} .
\end{aligned}
$$

Then, by using the Cauchy-Schwarz inequality and Lemma 2.2, the function $\mathcal{H}_{7}$ can be estimated as follows

$$
\begin{aligned}
\int_{0}^{t} \int_{\mathbb{R}}\left|\mathcal{H}_{7}(\tau, x)\right| \mathrm{d} x \mathrm{~d} \tau \leq & \frac{1}{4} \int_{0}^{t}\left\|\left(\varphi_{x}, \psi_{x}\right)(\tau)\right\|^{2} \mathrm{~d} \tau \\
& +C \int_{0}^{t}\left\|\left(\phi_{x}, z_{x}\right)(\tau)\right\|^{2}+\left\|\sqrt{V_{t}(\tau)}(\varphi, \phi)(\tau)\right\|^{2} \mathrm{~d} \tau .
\end{aligned}
$$

It follows from (3.12) that for $|(\varphi, \psi, \phi)| \rightarrow 0$,

$$
\begin{gathered}
\left|\tilde{f}_{1}\right|=O\left(\left|\varphi \Theta_{x}\right|+\left|\phi V_{x}\right|+\left|\varphi \phi_{x}\right|+\left|\phi \varphi_{x}\right|+\left|\varphi \varphi_{x}\right|\right), \\
\left|\tilde{f}_{2}\right|=C\left(\left|\Theta_{x x}\right|+\left|\Theta_{x}\right|\left(\left|V_{x}\right|+\left|\Theta_{x}\right|\right)\right)+O\left(\left|U_{x} \varphi\right|+\left|U_{x} \phi\right|+\left|\phi \psi_{x}\right|+\left|\varphi \psi_{x}\right|\right) .
\end{gathered}
$$

Then, by using the Cauchy-Schwarz inequality and Lemma 2.2, we have

$$
\begin{aligned}
\int_{0}^{t} \int_{\mathbb{R}}\left|\mathcal{H}_{8}(\tau, x)\right| \mathrm{d} x \mathrm{~d} \tau \leq & \left(\frac{1}{4}+C N(t)\right) \int_{0}^{t}\left\|\left(\varphi_{x}, \psi_{x}\right)(\tau)\right\|^{2} \mathrm{~d} \tau \\
& +C\left(\int_{0}^{t}\left(\left\|\phi_{x}(\tau)\right\|^{2}+\left\|\sqrt{V_{t}(\tau)}(\varphi, \phi)(\tau)\right\|^{2}\right) \mathrm{d} \tau+\varepsilon^{1 / 4}\right) .
\end{aligned}
$$

Substituting the estimates of $\mathcal{H}_{j}, j=7,8$ into (3.13), and using the fact that $N(t)$ and $\varepsilon$ are small enough, we have

$$
\begin{aligned}
& \int_{0}^{t}\left\|\left(\varphi_{x}, \psi_{x}\right)(\tau)\right\|^{2} \mathrm{~d} \tau-C \int_{0}^{t}\left(\left\|\sqrt{V_{t}(\tau)}(\varphi, \phi)(\tau)\right\|^{2}+\left\|\left(\phi_{x}, z_{x}\right)(\tau)\right\|^{2}\right) \mathrm{d} \tau \\
\leq & C\left(\left\|\left(\varphi_{0}, \phi_{0}, \psi_{0 x}\right)\right\|^{2}+\left\|\left(\varphi, \phi, \psi_{x}\right)(t)\right\|^{2}+\varepsilon^{1 / 4}\right) .
\end{aligned}
$$

Similarly, apply $\partial_{x}$ to system (3.12), then multiply the second equation and the third equation by $-V \varphi_{x x}$ and $\frac{2 V^{2}}{R \Theta} \psi_{x x}$, respectively. Calculating their sum and integrating over $[0, t] \times \mathbb{R}$, we easily get the following estimate:

$$
\begin{aligned}
& \int_{0}^{t}\left\|\left(\varphi_{x x}, \psi_{x x}\right)(\tau)\right\|^{2} \mathrm{~d} \tau-C \int_{0}^{t}\left(\left\|\sqrt{V_{t}(\tau)}(\varphi, \phi)(\tau)\right\|^{2}+\left\|\left(\phi_{x}, z_{x}\right)(\tau)\right\|_{1}^{2}\right) \mathrm{d} \tau \\
& -C \int_{0}^{t}\left\|\left(\varphi_{x}, \psi_{x}\right)(\tau)\right\|^{2} \mathrm{~d} \tau \leq C\left(\left\|\left(\varphi, \phi, \psi_{x}\right)(0)\right\|_{1}^{2}+\left\|\left(\varphi, \phi, \psi_{x}\right)(t)\right\|_{1}^{2}+\varepsilon^{1 / 4}\right) .
\end{aligned}
$$

Then using the estimates (3.14), (3.15), we get the desired estimate (3.11) and thus finish the proof of Proposition 3.4. 
3.3. Elliptic estimates. Eventually, we show the elliptic estimates with which we get the $L^{2}\left(L^{2}\right)$ estimates of the derivatives of $\phi$, and the $L^{\infty}\left(L^{2}\right)$ estimates of $z$ and its derivatives; thus the proof of Proposition 2.4 will be complete. We begin with the $L^{2}\left(L^{2}\right)$ estimates of the derivatives of $\phi$, i.e.

Lemma 3.5. Under the assumptions stated in Proposition 2.4, there holds

$$
\begin{aligned}
& \int_{0}^{t}\left\|\phi_{x}(\tau)\right\|_{1}^{2} \mathrm{~d} \tau \\
\leq & C\left(\int_{0}^{t}\left(\|z(\tau)\|_{2}^{2}+\left\|\sqrt{V_{t}(\tau)}(\varphi, \phi)(\tau)\right\|^{2}\right) \mathrm{d} \tau+\varepsilon \int_{0}^{t}\left\|\varphi_{x}(\tau)\right\|_{1}^{2} \mathrm{~d} \tau+\varepsilon^{1 / 4}\right) .
\end{aligned}
$$

Proof. Multiply the fourth equation in (2.4) by $\phi_{x}$, and then integrate the equality over $[0, t] \times \mathbb{R}$. We get

$$
\begin{aligned}
\int_{0}^{t} \int_{\mathbb{R}} 4 \Theta^{3} \phi_{x}^{2} \mathrm{~d} x \mathrm{~d} \tau= & \int_{0}^{t} \int_{\mathbb{R}} \phi_{x}\left(\left(\frac{z_{x}}{v}\right)_{x}-v z-\left(4 \Theta^{3}\right)_{x} \phi+\frac{\left(\Theta^{4}\right)_{x}}{V} \varphi\right) \mathrm{d} x \mathrm{~d} \tau \\
& +\int_{0}^{t} \int_{\mathbb{R}}-\phi_{x}\left\{\left(\frac{\left(\Theta^{4}\right)_{x}}{V}\right)_{x} \frac{1}{v}\right\}_{x} \mathrm{~d} x \mathrm{~d} \tau \\
& +\int_{0}^{t} \int_{\mathbb{R}}-\phi_{x}\left\{6 \Theta^{2} \phi^{2}+4 \Theta \phi^{3}+\phi^{4}\right\}_{x} \mathrm{~d} x \mathrm{~d} \tau .
\end{aligned}
$$

By the Cauchy-Schwarz inequality, the first term in the right-hand side can be estimated as

$$
\nu \int_{0}^{t}\left\|\phi_{x}(\tau)\right\|^{2} \mathrm{~d} \tau+\frac{C}{\nu} \int_{0}^{t}\left(\|z(\tau)\|_{2}^{2}+\left\|\sqrt{V_{t}(\tau)}(\varphi, \phi)(\tau)\right\|^{2}\right) \mathrm{d} \tau
$$

where $\nu$ is a small constant to be chosen later. Then to the second term in the right-hand side of (3.16), we have

$$
\int_{0}^{t} \int_{\mathbb{R}}-\phi_{x}\left\{\left(\frac{\left(\Theta^{4}\right)_{x}}{V}\right)_{x} \frac{1}{v}\right\}_{x} \mathrm{~d} x \mathrm{~d} \tau=\mathbb{F}_{1}+\mathbb{F}_{2},
$$

with

$$
\begin{aligned}
\mathbb{F}_{1} & :=\int_{0}^{t} \int_{\mathbb{R}}-\phi_{x}\left\{\left(\frac{\left(\Theta^{4}\right)_{x}}{V}\right)_{x x} \frac{1}{v}-\left(\frac{\left(\Theta^{4}\right)_{x}}{V}\right)_{x} \frac{V_{x}}{v^{2}}\right\} \mathrm{d} x \mathrm{~d} \tau \\
& \leq \varepsilon \int_{0}^{t}\left\|\phi_{x}(\tau)\right\|^{2} \mathrm{~d} \tau+\frac{C}{\varepsilon} \int_{0}^{t}\left\|\left\{\left(\frac{\left(\Theta^{4}\right)_{x}}{V}\right)_{x x} \frac{1}{v}-\left(\frac{\left(\Theta^{4}\right)_{x}}{V}\right)_{x} \frac{V_{x}}{v^{2}}\right\}\right\|^{2} \mathrm{~d} \tau \\
& \leq \varepsilon \int_{0}^{t}\left\|\phi_{x}(\tau)\right\|^{2} \mathrm{~d} \tau+C \varepsilon^{1 / 4} \\
\mathbb{F}_{2} & :=\int_{0}^{t} \int_{\mathbb{R}} \frac{\varphi_{x} \phi_{x}}{v^{2}}\left(\frac{\left(\Theta^{4}\right)_{x}}{V}\right)_{x} \mathrm{~d} x \mathrm{~d} \tau \leq C \varepsilon \int_{0}^{t}\left\|\left(\varphi_{x}, \phi_{x}\right)(\tau)\right\|^{2} \mathrm{~d} \tau .
\end{aligned}
$$

In the estimate of $\mathbb{F}_{1}$, we used the inequality $\int_{0}^{t} \|\left\{\left(\left(\Theta^{4}\right)_{x} / V\right)_{x x} \|^{2}+\right.$ $\left.\left\|\left(\left(\Theta^{4}\right)_{x} / V\right)_{x} V_{x}\right\|^{2}\right\} \mathrm{d} \tau \leq C \varepsilon^{3}$. Hence we get the estimate of the second term in the right-hand side of (3.16):

$$
C\left\{\varepsilon \int_{0}^{t}\left\|\left(\varphi_{x}, \phi_{x}\right)(\tau)\right\|^{2} \mathrm{~d} \tau+\varepsilon^{1 / 4}\right\}
$$


with the numerical constant $C$ independent of $\varepsilon$. Finally the third integral can be estimated as

$$
\begin{aligned}
& C \int_{0}^{t} \int_{\mathbb{R}}\left|\phi_{x}\right|\left[\left|\phi_{x}\right|\left(|\phi|+\left|\phi^{2}\right|+\left|\phi^{3}\right|\right)+\left|\Theta_{x}\right|\left(\left|\phi^{2}\right|+\left|\phi^{3}\right|\right)\right] \mathrm{d} x \mathrm{~d} \tau \\
\leq & C N(t) \int_{0}^{t}\left(\left\|\phi_{x}(\tau)\right\|^{2}+\left\|\sqrt{V_{t}(\tau)}(\varphi, \phi)(\tau)\right\|^{2}\right) \mathrm{d} \tau .
\end{aligned}
$$

Insert the above estimates into the equality (3.16), choosing $\nu \leq 2 \inf \Theta^{3}$; by using the fact $N(t)$ and $\varepsilon$ are sufficiently small, we have

$$
\begin{aligned}
& \int_{0}^{t}\left\|\phi_{x}(\tau)\right\|^{2} \mathrm{~d} \tau \\
\leq & C\left(\int_{0}^{t}\left(\|z(\tau)\|_{2}^{2}+\left\|\sqrt{V_{t}(\tau)}(\varphi, \phi)(\tau)\right\|^{2}\right) \mathrm{d} \tau+\varepsilon \int_{0}^{t}\left\|\varphi_{x}(\tau)\right\|^{2} \mathrm{~d} \tau+\varepsilon^{1 / 4}\right) .
\end{aligned}
$$

Next, we apply $\partial_{x}$ to the fourth equation in (2.4), multiply the result by $\phi_{x x}$, and then integrate the resulting equation over $[0, t] \times \mathbb{R}$ to obtain

$$
\begin{aligned}
\int_{0}^{t} \int_{\mathbb{R}} 4 \Theta^{3} \phi_{x x}^{2} \mathrm{~d} x \mathrm{~d} \tau= & \int_{0}^{t} \int_{\mathbb{R}} \phi_{x x}\left(4 \Theta^{3}\right)_{x} \phi_{x} \mathrm{~d} x \mathrm{~d} \tau \\
& +\int_{0}^{t} \int_{\mathbb{R}} \phi_{x x}\left(\left(\frac{z_{x}}{v}\right)_{x}-v z-\left(4 \Theta^{3}\right)_{x} \phi+\frac{\left(\Theta^{4}\right)_{x}}{V} \varphi\right)_{x} \mathrm{~d} x \mathrm{~d} \tau \\
& +\int_{0}^{t} \int_{\mathbb{R}}-\phi_{x x}\left\{\left(\frac{\left(\Theta^{4}\right)_{x}}{V}\right)_{x} \frac{1}{v}\right\}_{x x} \mathrm{~d} x \mathrm{~d} \tau \\
& +\int_{0}^{t} \int_{\mathbb{R}}-\phi_{x x}\left\{6 \Theta^{2} \phi^{2}+4 \Theta \phi^{3}+\phi^{4}\right\}_{x x} \mathrm{~d} x \mathrm{~d} \tau
\end{aligned}
$$

Now we are going to estimate each term in the right-hand side of the above equality. Firstly apply the Cauchy-Schwarz inequality to obtain

$$
\int_{0}^{t} \int_{\mathbb{R}} \phi_{x x}\left(4 \Theta^{3}\right)_{x} \phi_{x} \mathrm{~d} x \mathrm{~d} \tau \leq C \varepsilon \int_{0}^{t}\left\|\phi_{x}(\tau)\right\|_{1}^{2} \mathrm{~d} \tau
$$

and

$$
\int_{0}^{t} \int_{\mathbb{R}} \phi_{x x}\left(\left(\frac{z_{x}}{v}\right)_{x}-v z\right)_{x} \mathrm{~d} x \mathrm{~d} \tau \leq \nu \int_{0}^{t}\left\|\phi_{x x}\right\|^{2} \mathrm{~d} \tau+\frac{C}{\nu} \int_{0}^{t}\|z(\tau)\|_{3}^{2} \mathrm{~d} \tau
$$

where $\nu$ denotes a small constant to be determined later. Then

$$
\begin{aligned}
& \int_{0}^{t} \int_{\mathbb{R}} \phi_{x x}\left(-\left(4 \Theta^{3}\right)_{x} \phi+\frac{\left(\Theta^{4}\right)_{x}}{V} \varphi\right)_{x} \mathrm{~d} x \mathrm{~d} \tau \\
\leq & C \int_{0}^{t} \int_{\mathbb{R}}\left|\phi_{x x}\right|\left(\left|\Theta_{x x}\right|+\left|\Theta_{x}\right|^{2}\right)(|\phi|+|\varphi|) \mathrm{d} x \mathrm{~d} \tau+C \int_{0}^{t} \int_{\mathbb{R}}\left|\phi_{x x}\right|\left|\Theta_{x}\right|\left(\left|\phi_{x}\right|+\left|\varphi_{x}\right|\right) \mathrm{d} x \mathrm{~d} \tau \\
\leq & C N(t)\left(\int_{0}^{t}\left\|\phi_{x x}(\tau)\right\|^{2} \mathrm{~d} \tau+\varepsilon^{1 / 4}\right)+\varepsilon \int_{0}^{t}\left(\left\|\phi_{x}(\tau)\right\|_{1}^{2}+\left\|\varphi_{x}\right\|^{2}\right) \mathrm{d} \tau .
\end{aligned}
$$

As in our estimate of $\phi_{x}$, we have

$$
\int_{0}^{t} \int_{\mathbb{R}}-\phi_{x x}\left\{\left(\frac{\left(\Theta^{4}\right)_{x}}{V}\right)_{x} \frac{1}{v}\right\}_{x x} \mathrm{~d} x \mathrm{~d} \tau \leq C\left\{\varepsilon \int_{0}^{t}\left(\left\|\phi_{x x}(\tau)\right\|^{2}+\left\|\varphi_{x}(\tau)\right\|_{1}^{2}\right) \mathrm{d} \tau+\varepsilon^{1 / 4}\right\},
$$


and

$$
\int_{0}^{t} \int_{\mathbb{R}}-\phi_{x x}\left\{6 \Theta^{2} \phi^{2}+4 \Theta \phi^{3}+\phi^{4}\right\}_{x x} \mathrm{~d} x \mathrm{~d} \tau \leq C N(t) \int_{0}^{t}\left\|\phi_{x}(\tau)\right\|_{1}^{2} \mathrm{~d} \tau+C \varepsilon^{1 / 4} .
$$

Applying the above estimates to the equality, choosing $\nu \leq 2 \inf \Theta^{3}$, and using the fact $N(t)$ and $\varepsilon$ are sufficiently small, we have

$$
\begin{aligned}
& \int_{0}^{t}\left\|\phi_{x x}(\tau)\right\|^{2} \mathrm{~d} \tau \\
\leq & C\left(\int_{0}^{t}\|z(\tau)\|_{3}^{2} \mathrm{~d} \tau+\varepsilon^{1 / 4}+\varepsilon \int_{0}^{t}\left\|\varphi_{x}(\tau)\right\|_{1}^{2} \mathrm{~d} \tau+(N(t)+\varepsilon) \int_{0}^{t}\left\|\phi_{x}(\tau)\right\|^{2} \mathrm{~d} \tau\right) .
\end{aligned}
$$

Thus, together with estimate (3.17), we get the desired estimate and complete the proof.

It remains to show the $L^{\infty}\left(L^{2}\right)$ estimates of the radiative variable $z$ and its derivatives. We have the following Lemma.

Lemma 3.6. Under the assumptions stated in Proposition 2.4, there holds

$$
\|z(t)\|_{3}^{2} \leq C\left(\|(\varphi, \phi)(t)\|_{2}^{2}+\varepsilon^{1 / 4}\right)
$$

Proof. We multiply the equation satisfied by $z$,

$$
v z^{2}+\frac{z_{x}^{2}}{v}+\{\cdots\}_{x}=\frac{\left(\Theta^{4}\right)_{x}}{V} \varphi z+\left(\frac{\left(\Theta^{4}\right)_{x}}{V}\right)_{x} \frac{z_{x}}{v}+\left(\theta^{4}-\Theta^{4}\right) z_{x} .
$$

Then, we integrate the above equality with respect to $x$ and recal that $\{\cdots\}_{x}$ denotes the terms that disappear after integration. Using the Cauchy-Schwarz inequality, we have

$$
\|z(t)\|_{1}^{2} \leq C\left(\|(\varphi, \phi)(t)\|^{2}+\varepsilon^{1 / 4}\right)
$$

Next we apply $\partial_{x}$ to the elliptic equation and have

$$
v z_{x}-\left(\frac{z_{x x}}{v}\right)_{x}+\frac{z_{x}}{v^{2}} v_{x}+v_{x} z=\left(\varphi \frac{4 \Theta^{3} \Theta_{x}}{V}\right)_{x}-\left\{\left(\frac{\left(\Theta^{4}\right)_{x}}{V}\right)_{x} \frac{1}{v}+\left(\theta^{4}-\Theta^{4}\right)\right\}_{x x} .
$$

On the one hand, we multiply the last equation by $z_{x}$, then integrate the resulting equation with respect to $x$ to obtain

$$
\begin{aligned}
\left\|z_{x}(t)\right\|_{1}^{2} \leq & C \int_{\mathbb{R}}\left|v_{x}\right|\left(\left|z_{x}\right|^{2}+\left|z_{x} z_{x x}\right|\right) \mathrm{d} x+\int_{\mathbb{R}}\left|z_{x x}\right|\left|\varphi \frac{4 \Theta^{3} \Theta_{x}}{V}\right| \mathrm{d} x \\
& +\int_{\mathbb{R}}\left|z_{x x}\right|\left|\left\{\left(\frac{\left(\Theta^{4}\right)_{x}}{V}\right)_{x} \frac{1}{v}+\left(\theta^{4}-\Theta^{4}\right)\right\}_{x}\right| \mathrm{d} x .
\end{aligned}
$$

Note that $\left|v_{x}\right| \leq C(N(t)+\varepsilon)$ by the Sobolev inequality and the properties satisfied by the smooth approximation of the rarefaction wave; see Lemma 2.2. Then apply the Cauchy-Schwarz inequality to obtain

$$
\left\|z_{x}(t)\right\|_{1}^{2} \leq C\left(\|z(t)\|^{2}+\|\phi(t)\|_{1}^{2}+\|\varphi(t)\|^{2}+\varepsilon^{1 / 4}\right) .
$$


The details are omitted. On the other hand, we multiply (3.20) by $z_{x x x}$. Integrating the resulting equality with respect to $x$, we have

$$
\begin{aligned}
\left\|z_{x x}(t)\right\|^{2} \leq & C \int_{\mathbb{R}}\left|z_{x x x}\right|\left(\left|v z_{x}\right|+\left|v_{x} z\right|+\left|z_{x x} v_{x}\right|+\left|z_{x} v_{x x}\right|\right) \mathrm{d} x \\
& \left.+\int_{\mathbb{R}}\left|z_{x x x}\right| \mid\left\{\left(\frac{\left(\Theta^{4}\right)_{x}}{V}\right)_{x} \frac{1}{v}+\left(\theta^{4}-\Theta^{4}\right)\right\}\right\}_{x x} \mid \mathrm{d} x \\
& +\int_{\mathbb{R}}\left|z_{x x x}\right|\left|\left(\varphi \frac{4 \Theta^{3} \Theta_{x}}{V}\right)_{x}\right| \mathrm{d} x .
\end{aligned}
$$

Thus, by applying the Cauchy-Schwarz inequality, we have

$$
\left\|z_{x x x}(t)\right\|^{2} \leq C\left(\|z(t)\|_{2}^{2}+\|(\varphi, \phi)(t)\|_{2}^{2}+\varepsilon^{1 / 4}\right) .
$$

By multiplying (3.19) by a sufficiently large constant and adding the result to (3.21), we get the $L^{2}\left(H^{2}\right)$ estimate of $z$. Then we multiply the resulting estimate by a sufficiently large constant and add it to (3.22), obtaining the desired estimate (3.18) and completing the proof.

Acknowledgement. This work is a continuation of the $\mathrm{PhD}$ thesis of the author. The author would like to thank his supervisors Thierry Goudon and Jean-François Coulombel for their encouragement and guidance. The author is very grateful to the referees for carefully reading the manuscript and proposing many valuable suggestions.

\section{REFERENCES}

[1] C.M. Dafermos, Hyperbolic conservation laws in continuum physics, Grundlehren der Mathematischen Wissenschaften [Fundamental Principles of Mathematical Sciences], SpringerVerlag, Berlin, second edition, 325, 2005.

[2] S. Jiang, G. Ni and W. Sun, Vanishing viscosity limit to rarefaction waves for the NavierStokes equations of one-dimensional compressible heat-conducting fluids, SIAM J. Math. Anal., 38(2), 368-384 (electronic), 2006.

[3] S. Kawashima and A. Matsumura, Asymptotic stability of traveling wave solutions of systems for one-dimensional gas motion, Commun. Math. Phys., 101(1), 97-127, 1985.

[4] S. Kawashima, A. Matsumura and K. Nishihara, Asymptotic behavior of solutions for the equations of a viscous heat-conductive gas, Proc. Japan Acad. Ser. A Math. Sci., 62(7), 249-252, 1986.

[5] S. Kawashima, Y. Nikkuni and S. Nishibata, The initial value problem for hyperbolic-elliptic coupled systems and applications to radiation hydrodynamics, Analysis of Systems of Conservation Laws (Aachen, 1997), Chapman \& Hall/CRC Monogr. Surv. Pure Appl. Math., Chapman \& Hall/CRC, Boca Raton, FL, 99, 87-127, 1999.

[6] S. Kawashima, Y. Nikkuni and S. Nishibata, Large-time behavior of solutions to hyperbolicelliptic coupled systems, Arch. Ration. Mech. Anal., 170(4), 297-329, 2003.

[7] S. Kawashima and S. Nishibata, Shock waves for a model system of the radiating gas, SIAM J. Math. Anal., 30(1), 95-117 (electronic), 1999.

[8] S. Kawashima and Y. Tanaka, Stability of rarefaction waves for a model system of a radiating gas, Kyushu J. Math., 58(2), 211-250, 2004.

[9] C. Lin, J.F. Coulombel and Th. Goudon, Shock profiles for non-equilibrium radiating gases, Phys. D, 218(1), 83-94, 2006.

[10] C. Lin, J.F. Coulombel and Th. Goudon, Asymptotic stability of shock profiles in radiative hydrodynamics, C. R. Math. Acad. Sci. Paris, 345(11), 625-628, 2007.

[11] T.P. Liu and Z.P. Xin, Nonlinear stability of rarefaction waves for compressible Navier-Stokes equations, Commun. Math. Phys., 118(3), 451-465, 1988.

[12] T. Luo, Asymptotic stability of planar rarefaction waves for the relaxation approximation of conservation laws in several dimensions, J. Differ. Equs., 133(2), 255-279, 1997.

[13] A. Matsumura and K. Nishihara, Asymptotics toward the rarefaction waves of the solutions of a one-dimensional model system for compressible viscous gas, Japan J. Appl. Math., 3(1), $1-13,1986$. 
[14] A. Matsumura and K. Nishihara, Global stability of the rarefaction wave of a one-dimensional model system for compressible viscous gas, Commun. Math. Phys., 144(2), 325-335, 1992.

[15] D. Mihalas and B.W. Mihalas, Foundations of Radiation Hydrodynamics, Oxford University Press, New York, 1984.

[16] K. Nishihara, T. Yang and H. Zhao, Nonlinear stability of strong rarefaction waves for compressible Navier-Stokes equations, SIAM J. Math. Anal., 35(6), 1561-1597 (electronic), 2004.

[17] K. Nishihara, H. Zhao and Y. Zhao, Global stability of strong rarefaction waves of the JinXin relaxation model for the p-system, Commun. Part. Differ. Equs., 29(9-10), 1607-1634, 2004.

[18] L. Ruan and J. Zhang, Asymptotic stability of rarefaction wave for hyperbolic-elliptic coupled system in radiating gas, Acta Math. Sci. Ser. B Engl. Ed., 27(2), 347-360, 2007.

[19] D. Serre, Systems of Conservation Laws. 1, Hyperbolicity, entropies, shock waves, Translated from the 1996 French original by I.N. Sneddon, Cambridge University Press, Cambridge, 1999.

[20] J. Smoller, Shock Waves and Reaction-Diffusion Equations, Grundlehren der Mathematischen Wissenschaften [Fundamental Principles of Mathematical Sciences], Springer-Verlag, New York, second edition, 258, 1994.

[21] Z.P. Xin, Asymptotic stability of rarefaction waves for $2 * 2$ viscous hyperbolic conservation laws, J. Differ. Equs., 73(1), 45-77, 1988.

[22] Ya.B. Zeldovich and Yu.P. Raizer, Physics of Shock Waves and High-temperature Hydrodynamic Phenomena, Academic Press, 1966.

[23] H. Zhao, Nonlinear stability of strong planar rarefaction waves for the relaxation approximation of conservation laws in several space dimensions, J. Differ. Equs., 163(1), 198-222, 2000. 